\title{
Cross contamination of blood cultures associated with a multiple use venting device
}

\author{
R E Holliman, J D Johnson
}

\begin{abstract}
Aim-To investigate cross contamination of blood cultures associated with multiple use venting devices, which are widely used in clinical microbiology laboratories to reduce labour costs.

Methods-Systematic analysis of 13880 blood culture results in a large teaching hospital where multiple use venting devices were employed.

Results-Nine series of potential cross contamination were identified in a 12 month period. Four series involved coagulase negative staphylococci and were unlikely to represent true cross contamination. Five series involved blood cultures which had significant bacterial growth at the time of venting.

Conclusions-Multiple use venting devices can be associated with cross contamination of blood cultures. This may result from contamination of the internal lumen of the venting device which is not exposed to the biocide. Medical microbiologists should consider the possibility of cross contamination associated with venting procedures when interpreting blood culture results. Further development of multiple use venting devices is required to reduce the risk of cross contamination of cultures.

(F Clin Pathol 1999;52:231-233)
\end{abstract}

Keywords: blood culture; cross contamination; venting device

Blood culture remains an integral component of the investigation of sepsis, and optimal application of positive results improves patient care. ${ }^{12}$ Increasingly, clinical laboratories have adopted automated systems for blood culture. Some automated systems require transient venting of aerobic blood culture bottles to enhance the recovery of aerobic and microaerophilic organisms. Single use venting needles require significant manipulation by a technician and represent a potential safety hazard to the operator. Semiautomated devices have been introduced in an attempt to address these issues. Owing to the increased cost of materials some of these devices are designed for multiple use, introducing the potential for cross contamination of cultures by carry over of organisms from one bottle to another. We report our experience of blood culture cross contamination associated with a multiple use venting device.

\section{Methods}

BLOOD CULTURE

Blood from patients with suspected sepsis was cultured using the BacT/Alert system (Organon Teknika, Cambridge, UK), according to the manufacturer's instructions. On receipt in the clinical laboratory all aerobic blood culture bottles were transiently vented using the Steri/ VENT device, allowing filtered air to enter the bottle head space. The Steri/VENT device (Sterimatic, Stroud, Gloucestershire, UK) consists of two component parts, a Steri/ VENT and a Stericap. The Steri/VENT comprises a spring loaded, hollow bore needle which is used to pierce the diaphragm of the blood culture bottle. The vacuum within the blood culture bottle draws filtered air in through this needle. The Stericap is designed to sterilise the device before and after insertion into a blood culture by a minimum of 10 seconds' exposure of the external surface of the needle to a biocide. This biocide is effective for a minimum of 40 venting actions or for 24 hours after opening, whichever is the sooner. The colorimetric sensor on each blood culture bottle was checked before venting. Standard operating procedures require that bottles with positive sensors were not vented or loaded onto the machine but subject to immediate subculture and microscopy.

POSITIVE BLOOD CULTURES

Blood cultures found positive by inspection of the sensor or signalling positive on the blood culture machine were examined by Gram stain. Microscopy findings were reviewed by the duty medical microbiologist and then discussed with the clinical team caring for that patient. Subsequently, culture and antimicrobial susceptibility findings were reviewed and discussed in a similar manner. Initial and modified management of each patient was based on a consideration of all available laboratory and clinical findings and followed the hospital's written antibiotic policy.

\section{DETECTION OF POTENTIAL CROSS}

CONTAMINATION

Blood culture results over a 12 month period, May 1997 to April 1998, were reviewed for evidence of cross contamination in the laboratory by interrogation of the BacT/Alert computer database and clinical records for each patient.

Criteria for identifying potential cross contamination were as follows:

- Two or more blood culture isolates taken from different patients with the same species identified; 
Table 1 Potential cross contamination of blood cultures

\begin{tabular}{|c|c|c|c|c|c|}
\hline Series & Organism & Typing & $\begin{array}{l}\text { Biomedical } \\
\text { scientist } \\
\text { performing } \\
\text { venting }\end{array}$ & $\begin{array}{l}\text { Number of } \\
\text { positive blood } \\
\text { culture bottles }\end{array}$ & Further details \\
\hline 1 & $\begin{array}{l}\text { Coagulase negative } \\
\text { staphylococcus }\end{array}$ & - & A & 2 & Sequential cultures \\
\hline 2 & $\begin{array}{l}\text { Coagulase negative } \\
\text { staphylococcus }\end{array}$ & - & $\mathrm{B}$ & 2 & $\begin{array}{l}\text { One negative culture separating } \\
\text { the two positive cultures }\end{array}$ \\
\hline 3 & $\begin{array}{l}\text { Coagulase negative } \\
\text { staphylococcus }\end{array}$ & - & $\mathrm{C}$ & 2 & $\begin{array}{l}\text { One negative culture separating } \\
\text { the two positive cultures }\end{array}$ \\
\hline 4 & Klebsiella aerogenes & $\mathrm{K} 11, \mathrm{~K} 21$ & $\mathrm{D}$ & 2 & Sequential cultures \\
\hline 5 & $\begin{array}{l}\text { Coagulase negative } \\
\text { staphylococcus }\end{array}$ & - & $\mathrm{D}$ & 2 & $\begin{array}{l}\text { Three negative cultures separating } \\
\text { the positive cultures; one patient } \\
\text { on vancomycin and one on } \\
\text { gentamicin }\end{array}$ \\
\hline 6 & Enterococcus faecalis & $\begin{array}{l}\text { Identical SmaI digest } \\
\text { electrophoresis patterns }\end{array}$ & $\mathrm{E}$ & 2 & Sequential cultures \\
\hline 7 & Klebsiella aerogenes & $\mathrm{K} 52$ & $\mathrm{E}$ & 3 & Sequential cultures \\
\hline 8 & Streptococcus pneumoniae & Serogroup 1 & $\mathrm{~F}$ & 8 & $\begin{array}{l}\text { Sequence of } 11 \text { cultures: three } \\
\text { negative cultures within the series; } \\
\text { one taken from a patient on } \\
\text { vancomycin treatment }\end{array}$ \\
\hline 9 & Enterobacter sakazakii & Serotype 23 , biotype 22 & $\mathrm{~F}$ & 3 & Sequential cultures \\
\hline
\end{tabular}

- Five or less aerobic bottles vented between the two positive blood cultures;

- Identical antimicrobial susceptibility pattern for each isolate in a series.

Isolates from potential incidents of cross contamination were sent to an appropriate reference laboratory for confirmation of identity and typing. Laboratory records were used to document the biomedical scientists involved in the processing of each blood culture. The blood culture system computer database was interrogated to determine the initial reading for each culture bottle in each series at loading as well as the subsequent growth curve.

\section{ASSESSMENT OF BLOOD CULTURE BOTTLE} SENSORS

After completion of the investigation of potential cross contamination, a study of the performance of the blood culture bottle sensors was undertaken. These colorimetric sensors, mounted at the bottom of each bottle, change from green (no $\mathrm{CO}_{2} /$ no bacterial growth) to yellow $\left(\mathrm{CO}_{2}\right.$ production/bacterial growth). During a two week period in July 1998 the colour of every sensor was each recorded by two operators on arrival in the laboratory and on removal from the blood culture machine when signalling positive. Each sensor was classified as positive (yellow), equivocal (green/yellow), or negative (green).

\section{Results}

In all, 13880 blood culture sets were received over the 12 month period, of which 1971 sets (2792 bottles) produced a microbial isolate. A median of seven positive blood cultures were processed each day (range 0-20). Nine series of potential cross contamination were identified (table 1). Six different biomedical scientists had been responsible for processing these blood culture bottles. Five series comprised only sequential positive blood culture bottles while the remaining four series included negative cultures. Of the eight negative cultures within potential cross contamination series, three were taken from patients receiving treatment with antibiotics active against the relevant bacterium.
Examination of the blood culture system computer database showed that potential cross contamination series $1,2,3$, and 5 each began with a blood culture bottle which indicated negative on initial loading onto the machine, with an initial lag phase before subsequent growth. These four series involved isolates of coagulase negative staphylococci. In contrast, the initial cultures in series $4,6,7,8$, and 9 had markedly high readings when first loaded.

A further 509 blood culture sets (946 bottles) were studied during the assessment of sensor performance. Of these, two sets $(0.4 \%)$ - comprising four bottles-had yellow (positive) sensors on arrival in the laboratory. Subsequently 50 sets (64 bottles) signalled positive on the blood culture machine. Of these machine signal positive bottles, 50 (78\%) had positive, seven (11\%) equivocal, and seven (11\%) negative sensors on removal.

All findings were brought to the attention of the relevant manufacturers and the Medical Device Agency.

\section{Discussion}

We identified nine distinct series of potential cross contamination of blood cultures in a 12 month period by systematic application of defined criteria. Of these nine series, four are unlikely to represent true cross contamination (series 1, 2, 3, and 5) owing to the small number of organisms in the cultures at the time of venting and loading onto the blood culture machine (as shown by analysis of the growth curve) and the failure to grow the relevant organism from other bottles within the series which contained no antibiotic active against that organism. In these four series the organisms isolated were coagulase negative staphylococci. Such organisms are one of the commonest isolates from blood cultures, ${ }^{3}$ reflecting clinically significant findings associated with device related infection or contamination of the culture with skin flora during venepuncture. ${ }^{4}$ Consequently the chance detection of coagulase negative staphylococci in a series of blood cultures is relatively high. The power to distinguish staphylococcal isolates by lack of coagulase activity and antibiotic susceptibility pat- 
tern, as undertaken in our study, is low. ${ }^{5}$ Advanced methods, such as restriction fragment length polymorphism or pyrolysis mass spectrophotometry, may have shown that these isolates represented different strains but such methods are expensive and not readily available in a clinical laboratory.

Five series $(4,6,7,8$, and 9) are likely to represent true cross contamination. These cultures formed $0.6 \%$ of our positive blood cultures and $0.1 \%$ of all blood cultures processed. Cross contamination of blood cultures could arise from defects in the venting device or faults in the operator procedure. Potential defects in the venting device include failure of biocide activity and intrinsic resistance of the blood culture isolate to the biocide. As different batches of venting device were used over the course of the study and several bacterial species were found in cross contaminated cultures, defects in the venting device are unlikely to have been responsible for our findings. Potential faults in operator procedure include:

- failure to check for culture bottles indicating positive growth on the sensor panel before venting;

- failure to sterilise the culture bottle septum adequately;

- leaving an inadequate period between venting operations so that the biocide did not have sufficient time to act;

- failure to replace the biocide with sufficient regularity.

It is notable that the incidents of likely cross contamination were all associated with venting and loading cultures with high readings. Consequently, contamination of the venting needle by aerosol or immersion may have occurred. The present design of the venting device exposes only the outer surface of the needle to the biocide so that contamination of the inner lumen may persist. Several different biomedical scientists had undertaken this process, showing that repeated error rather than poor practice by one operator was respon- sible for any non-compliance with standard operating procedures.

Only a small percentage of blood cultures $(0.4 \%)$ was signal positive on arrival in the laboratory, so that a high level of operator concentration was required to avoid venting and loading a bottle with a significant microbial growth, thereby delaying the diagnosis and risking cross contamination of other cultures. Our studies indicate that $22 \%$ of cultures containing an increased level of microbial growth are associated with a negative or equivocal sensor. Such cultures cannot be detected before venting but the risk of cross contamination may be lower than when a bottle with a markedly increased reading is vented.

Our findings show that employing multiple use venting devices can be associated with cross contamination of blood cultures. Strict adherence to standard operating procedures is required when using these devices, and checking the blood culture bottle sensor before venting should be regarded as a critical control point. Medical microbiologists should consider the possibility of cross contamination when interpreting blood culture results. Further development of multiple use venting devices should be undertaken to reduce the risk of carry over after inadvertent venting of blood cultures with a high level of bacterial growth. Modifications to address these limitations are currently in development by the manufacturer.

We are grateful to Organon Teknika for helpful discussion in the course of this study and to Miss Dawn Vallance for her expert secretarial assistance. Bacterial typing studies were performed at the Central Public Health Laboratory, London.

1 Holliman RE. The therapeutic impact of blood culture results. F Hosp Infect 1986;7:185-8

2 Arbo MD, Snydman DR. Influence of blood culture results on antibiotic choice in the treatment of bacteremia. Arch Intern Med 1994;154:2641-5.

3 Schonheyder HC, Hojbjerg T. The impact of the first notification of positive blood cultures on antibiotic therapy. APMIS 1995;103:37-44.

4 Jenkins SG. Approaches to antibiograms and formularybased antibiotic reporting. Clin Microbiol Newslett 1990;12: based

5 Chandrasekar PH, Brown WJ. Clinical issues of blood cultures. Arch Intern Med 1994;154:841-9. 